\title{
3D Volumetric Display using Passive Optical Scatterers
}

\author{
Shree K. Nayar* \\ Columbia University \\ New York, NY 10027
}

\author{
Vijay N. Anand ${ }^{\dagger}$ \\ Columbia University \\ New York, NY 10027
}

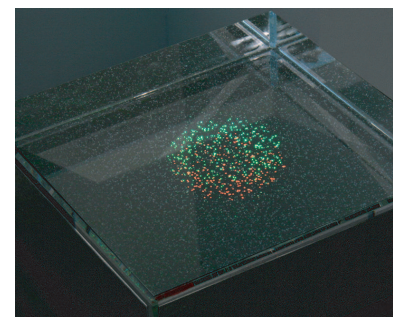

(a)

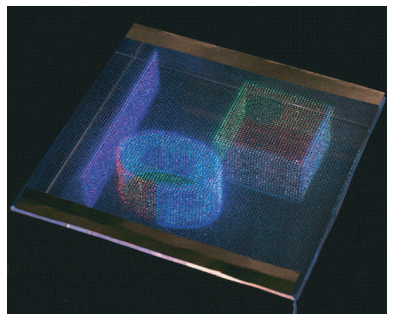

(b)

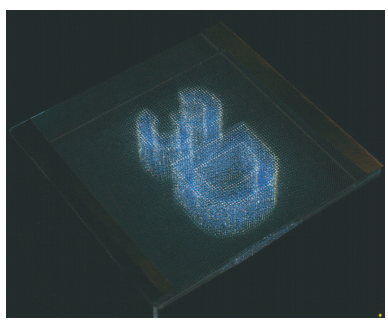

(c)

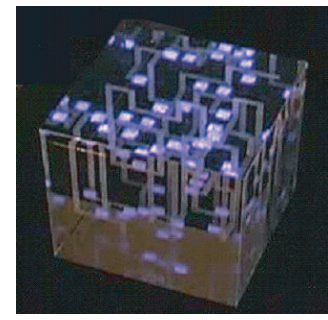

(d)

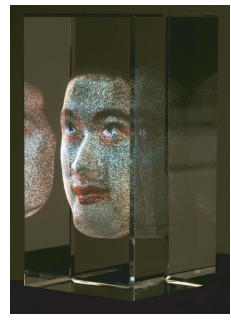

(e)

Figure 1: A class of low-cost volumetric displays are proposed that use a simple light engine and an indexable 3D point cloud made of passive optical scatterers. The point clouds are etched into a glass block using Laser Induced Damage (LID). Displays with different resolution characteristics have been developed that can render (a) true 3D objects, (b) extruded objects with arbitrary top layers and (c) purely extruded objects. The same approach has also been used to (d) extend the game Pac-Man to 3D and (e) create a 3D avatar.

\section{Approach}

The creation of a device that can display photorealistic 3D content at very high resolution may be considered a holy grail problem that has been pursued with great vigor over the past century. The goal of our work is less ambitious. It is to develop a very inexpensive class of volumetric displays [Okoshi 1976; Blundell and Schwartz 2000] that can present at relatively low resolution, and yet in a compelling way, certain types of $3 \mathrm{D}$ content. The types of content we wish to display are simple 3D objects, extruded objects and 3D surfaces that appear dynamic when projected with time-varying images. Our displays use a simple light engine and a dense cloud of passive optical scatterers. The basic idea is to trade-off the (2D) spatial resolution of the light engine to gain resolution in the third dimension. The simplest way to achieve such a trade-off is to use a stack of planar grids of scatterers where no two stacks overlap each other with respect to the projection rays of the light engine. However, such a semi-regular 3D grid suffers from poor visibility; as the viewer moves around the point cloud the fraction of points visible to the viewer varies dramatically and is very small for some of the viewing directions. Our key insight is to randomize the point cloud in a manner that is consistent with the projection geometry. We have verified that when a point cloud is randomized in a specific manner it produces a remarkably stable visibility function.

We have explored several ways of creating dense clouds of passive scatterers. We have chosen to use a technology called Laser Induced Damage (LID) that can very efficiently, precisely, and at a very low cost embed the desired point clouds in a solid block of glass. Each scatterer is a physical crack in the glass that is created by focusing a laser beam at the point. When a point in the cloud is lit by ambient light it is barely visible, but when it is lit by a focused source it glows brightly. We have studied the radiometric and spectral characteristics of LID scatterers and found that they have the properties needed to display objects in color and with sufficient brightness to be viewed within a 120 degree cone that is aligned with the projection direction. For illuminating the scatterers, we have developed an orthographic light engine that uses an off-the-shelf projector and inexpensive optics to create parallel rays with a large footprint. Orthographic projection enables us to use point clouds without resolution biases and makes the calibration of the display relatively straightforward.

*e-mail: nayar@cs.columbia.edu

†e-mail:vn2117@cs.columbia.edu

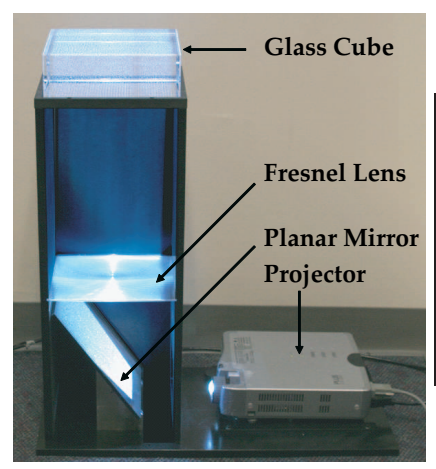

(a)

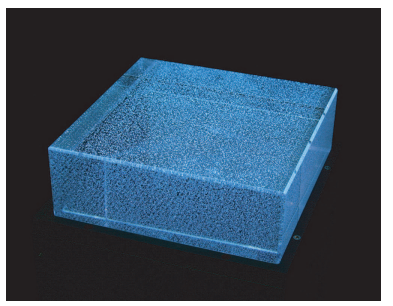

(b)
Figure 2: (a) An example implementation of the display system, including a light engine and an LID point cloud. (b) An example of a randomized LID cloud with $48 \times 48 \times 25$ points in a $200 \times 200 \times 70 \mathrm{~mm}$ glass cube, uniformly lit by a projector.

\section{Implementations}

Figure 2(a) shows the architecture of our display system. The system includes a projector-based light engine and an LID point cloud. Figure 2(b) shows an example of a randomized LID cloud with $48 \times 48 \times 25$ points in a $200 \times 200 \times 70 \mathrm{~mm}$ glass cube. We have developed several versions of our volumetric display, each one designed to meet the needs of a specific class of objects or a specific application. We have implemented point clouds with 10,000 points for the display of true 3D objects (see Figure 1(a)), 190,500 points for the display of extruded objects with arbitrarily textured top surfaces (see Figure 1(b)), 180,500 points for the display of purely extruded objects (see Figure 1(c)), 83,866 points for the extension of the game Pac-Man to 3D (see Figure 1(d)), and 127, 223 points for the face model used to create a 3D avatar (see Figure 1(e)).

\section{References}

Blundell, B. G., AND Schwartz, A. J. 2000. Volumetric Three Dimensional Display Systems. John Wiley and Sons, Inc.

Oкоsнi, T. 1976. Three-Dimensional Imaging Techniques. Academic Press, San Diego, CA. 